mouse gut bacteria were mainly composed of Fimicutes (Ruminococcaceae, Lachnospiraceae, Clostridiaceae) and Bacteroidetes (S24-7, Porphyromonadaceae). More specifically, Ruminococcus was significantly reduced in the three NAFLD models than the Control and was identified as the biomarker of NAFLD in LEfSe analysis. More biomarkers at genus level (Lachnospira, S24-7, etc.) were identified in pairwise comparison of one mice model with the Control.

Conclusions In summary, the composition of gut microbiota varied remarkably between mice administrated different experimental diets to induce non-alcoholic fatty liver disease.

\section{IDDF2018-ABS-0167 THE TCF3/MIR-145-5P/LINC00113 POSITIVE FEEDBACK LOOP PROMOTES COLORECTAL CANCER PROGRESSION BY SILENCING STAT1}

Changsheng Yan*. Zhongshan Hospital Xiamen University, China

\subsection{6/gutjnl-2018-IDDFabstracts.20}

Background To identify the role of LINC00113 in colorectal cancer.

Methods Jaspar and Cor $\mathrm{R}$ package was used to predict the potential translational factor of LINC00113. CHIP and dual luciferase report assay were enrolled to confirm the binding sites between LINC00113 and translational factor. Besides, dual luciferase report assay was used to confirm the binding site of LINC00113, miRNA and TCF3. Flow cytometry was used to detect the cell cycle and apoptosis. RIP assay was used to confirm the binding of LINC00113 and DNMT1.

Results Using the Gene expression omibus (GEO) data set and bioinformatics analyses, we identified LINC00113 as an independent risk prognostic factor in colorectal cancer. To identify the mechanism how LINC00113 in colorectal cancer, we firstly to seek the translational factor which could up-regulate the expression of LINC00113. We found that the expression level of TCF3 was strongly positively correlated with LINC0113. Besides, knockdown and overexpress of TCF3 could coordinately lead to the alteration of LINC00113. What's more, CHIP and luciferase assay confirm that TCF3 binds to the promoter sequence of LINC00113 and thus promoting the expression of LINC00113. Then we found that LINC00113 might be a negative regulator of miR-145-5 $\mathrm{p}$ by loss- and gain-of-function assay. Besides, we found that miR$149-5 \mathrm{p}$ had the potential ability binding to the 3'-UTR of TCF3, thus leading to the degradation of TCF3. However, as the increased level of LINC00113, the expression level of miR-145-5 p was lowly expressed in colorectal cancer, which leads to the expression level of TCF3 significantly up-regulated in colorectal cancer. At last, the high level of TCF3 in turn promotes the expression of LINC00113. Moreover, we also found that LINC00113 could bind to DNMT1 to promote the methylation of promoter sequence of STAT1 and leading to the down-regulation of STAT1, which act as a tumour suppressor in colorectal cancer.

Conclusions Our studies uncover a mechanism for constitutive LINC00113/miR-144-5 p/TCF3 feedback loop activation in colorectal cancer. Besides, high expressed of LINC00113 resulted in the down-regulation of STAT1, which further promoted the progression of colorectal cancer.

\section{IDDF2018-ABS-0176 ASSESSMENT OF QUALITY OF LIFE IN ADVANCED GASTRO-INTESTINAL CANCER PATIENTS RECEIVING CHEMOTHERAPY}

Ritu Balal ${ }^{*}$, Harminder Singh. BFUHS, Faridkot, India

\subsection{6/gutjnl-2018-IDDFabstracts.21}

Background The objective of this study is to assess the common gastro-intestinal (GI) symptoms and quality of life (QOL) in advanced GI tract cancer patients with the help of MD Anderson Symptom Inventory (MDASI-GI) validated scale. The objective of this study is to assess the common gastrointestinal (GI) symptoms and quality of life (QOL) in advanced GI tract cancer patients with the help of MD Anderson Symptom Inventory (MDASI-GI) validated scale.

Methods This is a cross-sectional, non-interventional and observational study. A total of 48 patients were interviewed about the GI symptoms and their effect on day to day life with MDASI-GI scale after taking written informed consent from patients.

Results The study population consisted of 23 males and 25 females with an average age of 48.16 years. Among cancer category, $52.08 \%$ were of Upper GI tract cancer. Symptomwise $59.12 \%$ showed mild category of GI symptoms. According to QOL, $65.12 \%$ of the patients mildly affected with reference to day to day activities. Out of a total of 48 patients, 25 had esophageal/upper GI cancer; 10 were of hepatobiliary followed by 8 having colorectal cancer; and 5 with buccal mucosal cancer. 25 patients were treated with Cisplatin/Oxaliplatin with 5-Fluorouracil chemotherapy; 11 patients received Gemcitabine; 8 were given Paclitaxel; and 4 received Methotrexate.

Abstract IDDF2018-ABS-0176 Table 1 Demographic data, type of cancer and symptoms

\begin{tabular}{lll}
\hline Parameters & Number & Percentage \\
\hline Male & 23 & 47.92 \\
Female & 25 & 52.08 \\
Age group (Years) & & \\
$41-50$ & 19 & 39.58 \\
$51-60$ & 07 & 14.58 \\
$61-70$ & 15 & 31.26 \\
$>70$ & 07 & 14.58 \\
Female History & & \\
Present & 09 & 18.75 \\
Absent & 39 & 81.25 \\
Type of Cancer: & & \\
Upper GI & 25 & 52.08 \\
Hepatobiliary & 10 & 20.84 \\
Colorectal & 08 & 16.67 \\
Buccal mucosa & 05 & 10.41 \\
Symptoms: & & \\
Mild (0-3) & & 59.12 \\
Moderate (4-7) & & 33.33 \\
Severe (8-10) & & 07.55 \\
Q0L & & \\
Mild (0-3) & & 11.75 \\
Moderate (4-7) & & \\
Severe (8-10) & & \\
\hline & & \\
\hline
\end{tabular}


Conclusions The result of this study shows that most of GI symptoms due to GI cancer were in mild to moderate category and can be managed with prior knowledge of their occurrence. Managing GI symptoms will not ameliorate the disease process but certainly will improve the daily activities and add to the improved quality of life.

\section{IDDF2018-ABS-0183 P53-INDUCED MIR-1249 SUPPRESSES TUMOUR PROGRESSION BY TARGETING VEGFA AND HMGA2 IN COLORECTAL CANCER}

Xiaoxiang Chen*, Shukui Wang. School of Medicine, Southeast University, China

10.1136/gutjnl-2018-IDDFabstracts.22

Background MicroRNAs (miRNAs) are an important class of functional regulators involved in human cancers development, including colorectal cancer (CRC). Exploring aberrantly expressed miRNAs may provide us with new insights into the initiation and development of CRC by functioning as oncogenes or tumour suppressors. The aim of our study is to discover the expression pattern of miR-1249 in CRC and investigate its clinical significance as well as a biological role in CRC progression.

Methods QRT-PCR was used to detect the relative expression of miR-1249 in CRC tissues and cell lines. EdU, CCK-8, Wound healing, transwell and HUVECs tube formation assays were performed to assess the effect of miR-1249 on CRC cell proliferation, migration, invasion and angiogenesis in vitro, nude mouse xenograft, tail vein injection and chicken chorioallantoic membrane(CAM) model were used to observe CRC growth, metastasis and angiogenesis in vivo. Luciferase reporter assay, western blot, immunohistochemistry(IHC) and
immunofluorescence(IF) staining were fulfilled to explore the molecular mechanism of miR-1249 in CRC.

Results MiR-1249 was found to be markedly downregulated in CRC tissues and cell lines. The expression of miR-1249 was negatively related to $\mathrm{pN}$ stage, $\mathrm{pM}$ stage, TNM stage and overall survival(OS). Moreover, we found that miR-1249 was a direct transcriptional target of P53 and revealed that P53-induced miR1249 inhibited tumour growth, metastasis and angiogenesis in vitro and vivo. Additionally, we verified that miR-1249 suppressed CRC proliferation and angiogenesis by targeting VEGFA as well as inhibited CRC metastasis by targeting VEGFA and HMGA2. Further studying showed that miR-1249 suppressed CRC cell proliferation, migration, invasion and angiogenesis via VEGFAmediated Akt/mTOR pathway as well as inhibited EMT process of CRC cells by targeting VEGFA and HMGA2.

Conclusions Our study demonstrated that P53-induced miR1249 might suppress CRC growth, metastasis and angiogenesis by targeting VEGFA and HMGA2, as well as regulate Akt/ mTOR pathway and EMT process in the initiation and development of CRC. miR-1249 might be a novel the therapeutic candidate target in CRC treatment.

\section{IDDF2018-ABS-0184 LNCRNA AGPG REGULATES ANABOLISM REMODELLING THROUGH AFFECTING PFKFB3 STABILITY IN ESCC}

${ }^{1}$ Jia Liu*, 'Ze-Xian Liu, ${ }^{1} Q i-N i a n$ Wu, ${ }^{2}$ Chau-Wei Wong, 'Yun-Xin Lu, ${ }^{1} Y$ Ying-Nan Wang, ${ }^{1}$ Yun Wang, ${ }^{1}$ Jia-Huan Lu, ${ }^{1}$ Hong-En Yu, ${ }^{1}$ Huai-Qiang Ju, 'Rui-Hua Xu. ${ }^{1}$ Sun Yat-sen University Cancer Center, State Key Laboratory of Oncology in South China, Collaborative Innovation Center for Cancer Medicine, China; ${ }^{2}$ The First Affiliated Hospital of Sun Yat-sen University, China

10.1136/gutjnl-2018-IDDFabstracts.23
A

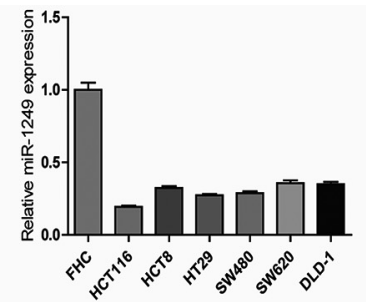

B

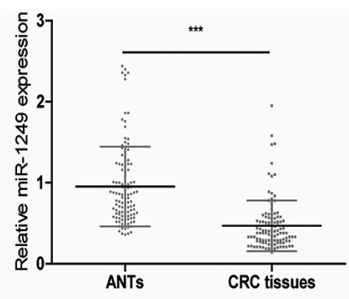

C

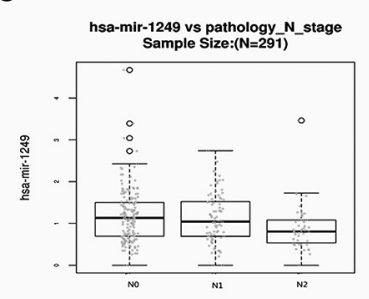

$\mathrm{F}$

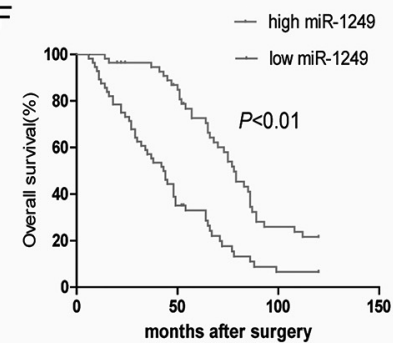

D

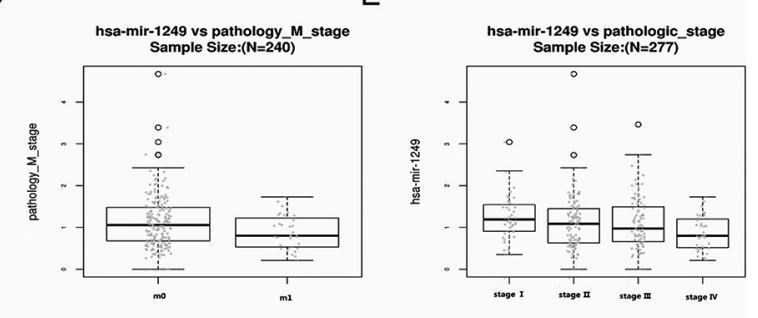

